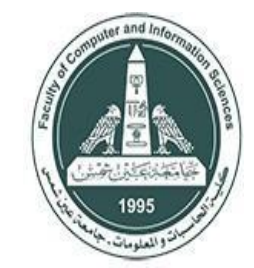

International Journal of Intelligent Computing and Information Sciences

\title{
DIAGNOSIS OF ALZHEIMER'S DISEASE BY THREE- DIMENSIONAL CONVOLUTIONAL NEURAL NETWORK USING UNSUPERVISED FEATURE LEARNING METHOD
}

\author{
Sarah A. Soliman* \\ Computer Science Department \\ Higher Technological Institute \\ Cairo, Egypt \\ sara_cs2003@hotmail.com
}

\author{
El-Sayed A. El-Dahshan \\ Computer Science Department \\ Egyptian E-Learning University, \\ Ain Shams University \\ Cairo, Egypt \\ e eldahshan@yahoo.com
}

\author{
Abdel-Badeeh M. Salem \\ Computer Science Department \\ Faculty of Computer and \\ information Sciences, Ain Shams \\ University \\ Cairo, Egypt \\ absalem@cis.asu.edu.eg
}

Received 2021- 6-13; Revised 2021-10-11; Accepted 2021-10-13

\begin{abstract}
The rise of Deep Learning in the past two decades has prompted research into solutions to help improve Alzheimer's diagnosis based on neuroimaging data. As such, a wide variety of different techniques have been used, but a clear turn towards the use of Convolutional Neural Networks (CNN) has been observed in the last decade. To effectively predicate Alzheimer's Disease (AD), this paper proposed a two stage method. The first stage involves learning the best representation of the training data using an improved sparse autoencoder (SAE), an unsupervised neural network. The second stage involves using a 3D-Convolutional Neural Network (3D-CNN) to differentiate between the health status and diseased status based on the learned records and MRI scan of the brain. The SAE was optimized so as to train an efficient model. We report on experiments using the ADNI data set involving 897 historical scans. We demonstrate that using $3 D$ convolutional neural networks with sparse auto encoder outperform several other classifiers stated in the literature.
\end{abstract}

Keywords: Alzheimer's disease (AD), Sparse autoencoder (SAE), 3D-Convolutional Neural Network (3D-CNN), Feature Learning.

\section{Introduction}

Alzheimer's disease (AD) is thought to be the most common type of dementia. Alzheimer's disease (AD) is a progressive neurological illness that causes memory loss and impairment in intellectual ability and other mental functions [1]. Although Alzheimer's disease is now incurable, it can be prevented from advancing if diagnosed

* Corresponding author: Sarah A. Soliman

Computer Science Department, Higher Technological Institute, Cairo, Egypt

E-mail address: $\underline{\text { sara_cs2003@hotmail.com }}$ 
early [2]. In 2006, 26.6 million persons were diagnosed with Alzheimer's disease. By 2050, Alzheimer's disease is expected to affect 1 in 85 persons worldwide, with around $43 \%$ of cases requiring high-level care [3]. The rising prevalence of Alzheimer's disease (AD) is causing alarm among clinicians, who perceive a pressing need for a reliable and effective computer-aided diagnostic (CAD) technique. Especially for those over the age of 65 who suffer from Alzheimer's disease. Several machine learning approaches have failed to diagnose Alzheimer's disease [4]. Due to workflow constraints, researchers have been forced to work with binary categories rather than numerous classes, despite the likely negative impacts on producing reliable judgments.

Magnetic resonance imaging (MRI), a technology that employs a magnetic field and radio waves to create a detailed 3D image of the brain, can help diagnose the condition early [5]. Advanced neuroimaging techniques, including as magnetic resonance imaging (MRI), have been developed and exploited to find structural biomarkers for Alzheimer's disease [6]. Integrating large-scale, high-dimensional multimodal neuroimaging data has become difficult due to the rapid advancement of neuroimaging technology. As a result, interest in computer-assisted machine learning methodologies for integrative analysis has exploded [7].

Deep learning approach has been able to categories, extract high level features, and will also help in the correct diagnosis of AD patients with less time due to the rapid development of machine learning algorithms [8]. Sparse auto encoders (SAE) have recently demonstrated outstanding performance in a variety of unsupervised machine learning applications. It has outstanding performance in learning good feature representations in complicated and huge datasets, making it a promising answer to the difficulty of AD prediction [9]. An auto encoder consists of two functions: an encoder that converts the original d-dimensional input data to an intermediate or hidden representation, and a decoder that converts the intermediate or hidden representation back to a d-dimensional vector that is as close as possible to the encoder's original input [10]. Furthermore, in recent years, 3D Convolutional Neural Networks (3DCNN) have exhibited huge achievements for image processing tasks [11] [12] [13]. 3D-CNN is a multi-layer artificial neuron deep feedforward neural network. CNNs [14] are used to automatically learn general features. CNNs are trained using a back propagation technique and typically comprise of several convolutional layers, pooling layers, and fully connected layers, with fully connected layers or other types of layers connecting to the output units. Because pixels share the convolution kernel, CNNs have fewer connections and smaller parameters, making them more widely used and easier to train [15].

In this paper, we proposed two stage methods to predicate AD. The first stage uses training sparse autoencoder (SAE) approach to perform unsupervised feature leaning and prediction of AD. We spot the light on developing a SAE model to learn effective features from the $\mathrm{AD}$ dataset and then perform classification using the learned features. The model is optimized using the adaptive moment estimation (Adam) algorithm to achieve dynamic adjustment of different parameters, and a batch normalization technique is applied to avoid overfitting and to improve the 
performance, speed, and stability of the model. The second stage involves using 3DConvolutional Neural Network (3D-CNN) to predict the health status based on the learned records.

This article is organized as follows. Section 2 discusses briefly the related work. Section 3 presents the proposed model. Section 4 reports the experimental details and the results. Finally, Section 5 gives conclusion of our work and the future work.

\section{Related work}

Through a review of prior studies on Alzheimer's disease diagnosis, it was determined that images with high dimension and a small sample size are one of the study's most significant problems. Deep learning methods, such as deep sparse multi-task learning [16], stacked auto-encoder [17], sparse regression models [18], sparse auto-encoder [19], and CNN, are the most recently used methods for feature selection.

The authors of ref [20] recommended two methods: first, learn the features that characterize Alzheimer's illness using sparse filtering learning. For automatic classification, a second SoftMax regression technique was trained. The goal of this study was to increase accuracy by reducing the effect of features with low information load and features with no information load in feature selection.

The 3D Capsule Network, 3D-Convolutional Neural Network, and pre-trained 3Dautoencoder were utilised to diagnose Alzheimer's disease early in [21]. A 3DCapsule Networks (CapsNets) can train quickly, even with little datasets, and can handle image rotations and transitions with ease. The goal of this research is to improve detection performance over Deep-CNN alone. The authors of ref [22] used a new framework based on deep learning methodologies for early diagnosis of Alzheimer's disease, which included stacked sparse auto-encoders and a softmax output layer. The goal of this technique was to simultaneously accomplish dimensionality reduction and data fusion. The binary classification provides a performance boost.

In [23], an unsupervised Convolutional Neural Networks technique for feature extraction was presented, followed by the use of an unsupervised predictor to reach a final diagnosis. As input data, the authors used two types of data forms: one slice and three orthogonal panels (TOP) of an MRI image. For one slice data and TOP data, this model has accuracy of 95.5 percent and 97 percent, respectively. A sparse auto encoder was created to detect Alzheimer's disease in (PET/CT) brain images in [24]. The classifier is trained in two stages: the first stage is supervised training to identify the $\mathrm{AD}$, and the second stage is unsupervised learning to provide an image output. The precision achieved 98.6 percent.

The authors of [25] attempted a new exploratory data analysis of Alzheimer's disease based on deep convolutional autoencoders, with the goal of discovering correlations between cognitive symptoms and the underlying neurodegenerative process. A deep convolutional autoencoder (CAE) architecture was presented. Two experiments were defined in this paper: classification using a linear support vector machine and 
prediction using a neural classifier based on an MLP with two hidden layers of 64 neurons and ReLU activations. The accuracy of this work was up to $84 \%$.

\section{Proposed Methodology}

Our methodology is decomposed into two phases. In, first phase we used sparse auto encoder as feature learning technique. In, second phase, the output from the previous phase used as the input to 3D-convolutional neural network model. (Show figure 1).

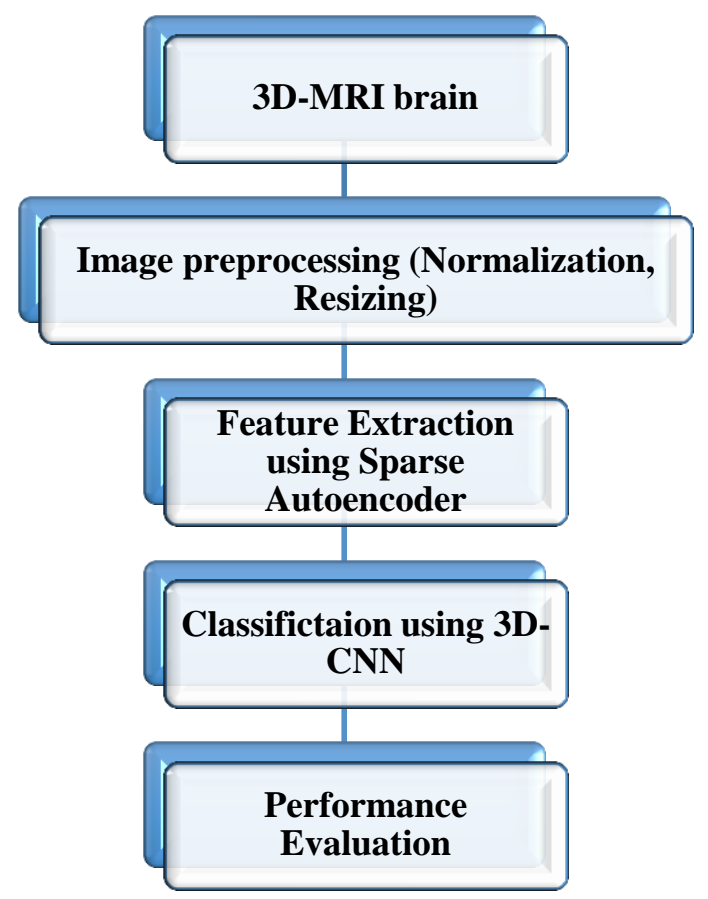

\subsection{Dataset}

Figure 1: The proposed Methodology

We used the structural brain MRI scans from the ADNI dataset (ADNI.LONI.USC.EDU). The ADNI was launched in 2003 as a public private partnership, led by Principal Investigator Michael W. Weiner, MD. The primary goal of ADNI has been to test whether serial MRI, positron emission tomography, other biological markers, and clinical and neuropsychological assessment can be combined to measure the progression of MCI and early AD. For up-to-date information, see WWW.ADNI-INFO.ORG [26]. A total of 897 subjects (297 patients with probable AD, 300 patients with MCI, and 300 healthy controls) were considered in this study (Table 1).

Table 1: Demographic Clinical features of AD and MCI patients and healthy controls from the ADNI

\begin{tabular}{|c|c|c|c|c|c|c|c|c|c|}
\hline Modality & $\begin{array}{l}\text { Total } \\
\text { subj. }\end{array}$ & Group & Subj. & Female & $\begin{array}{l}\text { Mean } \\
\text { of age }\end{array}$ & SD & Male & $\begin{array}{c}\text { Mean of } \\
\text { age }\end{array}$ & SD \\
\hline \multirow{3}{*}{ MRI } & \multirow{3}{*}{897} & $\mathrm{HC}$ & 300 & 157 & 78.72 & 4.4 & 143 & 79.72 & 5.25 \\
\hline & & MCI & 300 & 146 & 77.026 & 7.7 & 154 & 78.096 & 7.32 \\
\hline & & $\mathrm{AD}$ & 297 & 151 & 75.44 & 7.9 & 146 & 76.33 & 7.89 \\
\hline
\end{tabular}




\subsection{Data Preprocessing}

The data was merged into 3D as the size was $(256 \times 256 \times 48)$ Length Width and no. of scans for each patient to create a 3D image to feed to the model after the images were imported from the backup folder using Collaboratory framework, the resizing and normalisation processes were applied to all of the data before putting them in any classes, and then the data was merged into 3D as the size was $(256 \times 256 \times 48)$ Length Width and After completing this stage, a process of collecting random patches of data is started in order to obtain 1000 random patches of each photo of the patients we have and feed them into the model later to boost model dependability, maintain model control, and prevent bias.

\subsection{Sparse AutoEncoder (SAE)}

The approach utilized to implement the proposed sparse autoencoder is described in this section. A sort of unsupervised neural network design known as an autoencoder duplicates its input at the output. It is made up of two parts: an encoder and a decoder. The goal of AEs is to learn low-level representations of incoming data that are then deformed to project the original data. The encoder converts the input into a new format. The output decodes this new representation to reconstruct the input $\mathrm{x}^{\prime}$ using Equations (1) and (2), where $\mathrm{x}$ is the input and $\mathrm{z}$ is the new representation.

$$
\begin{aligned}
& Z=h(W x+b) \\
& X^{\prime}=g\left(W^{\prime} z+b^{\prime}\right)
\end{aligned}
$$

In the following formula, $\mathrm{h}$ represents the hidden layer activation function and $\mathrm{g}$ represents the output layer activation function, $\mathrm{W}$ and $\mathrm{W}^{\prime}$ represent weight matrices, and $b$ and $b$ represent the encoder and decoder bias vectors, respectively. Instead of using Relu, Tanh, and other activation functions, we used the sigmoid activation function shown in Equation (3).

$$
h=g=\frac{1}{1+e^{-x}}
$$

Our approach builds the reconstruction error function $\mathrm{E}$ between the input $\mathrm{x}$ and reconstructed input $x^{\prime}$ using mean absolute error (MAE), as stated in Equation (4).

A sparse autoencoder is created in this research in order to achieve an effective lowlevel representation of the input data under sparse limitations. As a result, regularisation is used to display sparsity in the cost function. The average activity of neurons in the buried layer is P i. (See Equation (5) for further information.)

$$
\widehat{P l}=\frac{1}{n} \sum_{j=1}^{n} z_{i}\left(x_{j}\right)
$$

$\mathrm{I}, \mathrm{n}$, and $\mathrm{j}$ indicate the ith neuron, total number of training samples, and $\mathrm{jth}$ training sample, respectively, in the preceding expression.

Furthermore, instead of using the standard stochastic gradient descent or other versions to train a resilient SAE, the Adam algorithm [13] is employed. The Adam 
optimization technique allows us to apply a different learning rate for distinct parameters and to do dynamic parameter adjustments. Algorithm 1 shows the approach for the proposed sparse autoencoder (See figure 2).

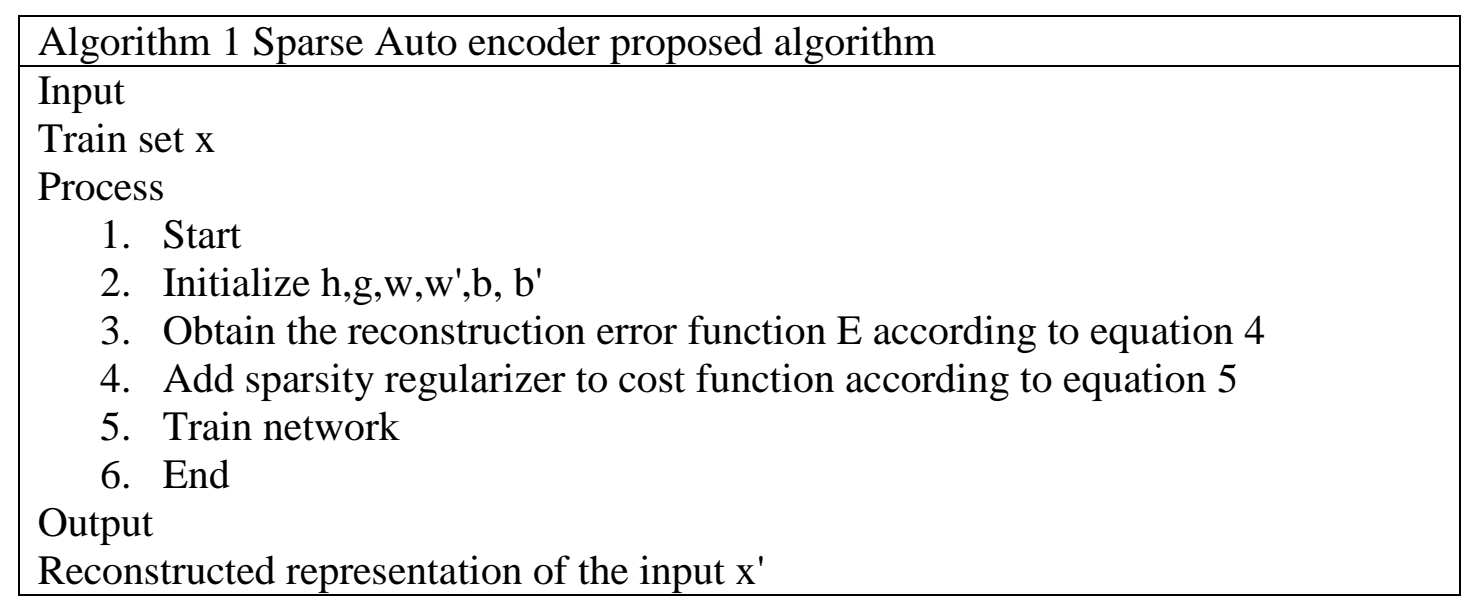

Figure 2: Sparse Auto-Encoder Proposed Algorithm

\section{- Training Sparse Autoencoder}

The sparse autoencoder model is a Sequential model consist of three layers: the first layer is the dense 1 layer of value 343, the second layer (dense 2) is with 410 with sigmoid activation function and the third layer (dense 3 ) with 343 with sigmoid activation function as show in figure 3. This autoencoder uses regularizers to hold the best weights for the current model to be used later on in another model as initial weights and to learn a sparse representation in the first layer. So, we can control the influence of these regularizers by setting various parameters: L2 Weight Regularization controls the impact of an L2 regularizer for the weights of the network.

In our approach, we train an autoencoder on a set of selected 3D objects extracted from the MRI scans. We extract 1000 random patches from 3888 scans in the training set .Adam optimizer was used to optimize the model output and Mean Absolute Error was used as loss function. The model succeeded in getting a loss of $23 \%$ which gave us an average of accuracy of $75 \%$ for this model.

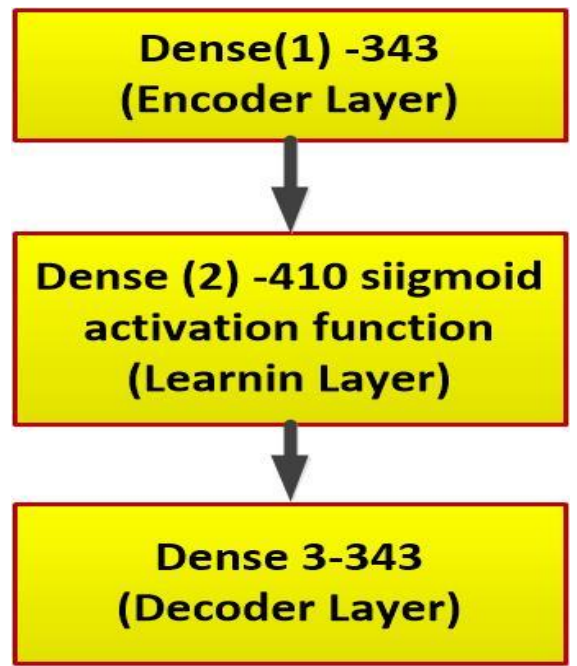

Figure 3: Block diagram of sparse autoencoder 


\subsection{Three-Dimensional Convolutional Neural Network}

CNNs are a sort of feedforward neural network that was created with the goal of processing images despite various distortions. In addition to the input and output layers, standard CNNs typically alternate convolutional and max-pooling layers, followed by a small number of fully-connected layers, as shown in Fig. 4. The first neural layer to receive an input signal is the convolutional layer, where neurons detect the primary features that characterize the images and store the information in a 'feature map' that shows the link between the neurons and their features. It is customary to apply a nonlinear layer immediately after each convolutional layer (or activation layer). This layer, which simply sets all negative activations to 0 , improves the model's nonlinear properties and the overall network's nonlinear properties without changing the convolutional layer's receptive fields. Following that is the pooling layer, which performs a spatial downsampling procedure. The fully linked layers are the last layers in the network, with neurons connected to all neurons from the previous layer. The amount of parameters that must be learned is reduced thanks to $\mathrm{CNN}$ properties, which improves training performance for general deep learning algorithms.

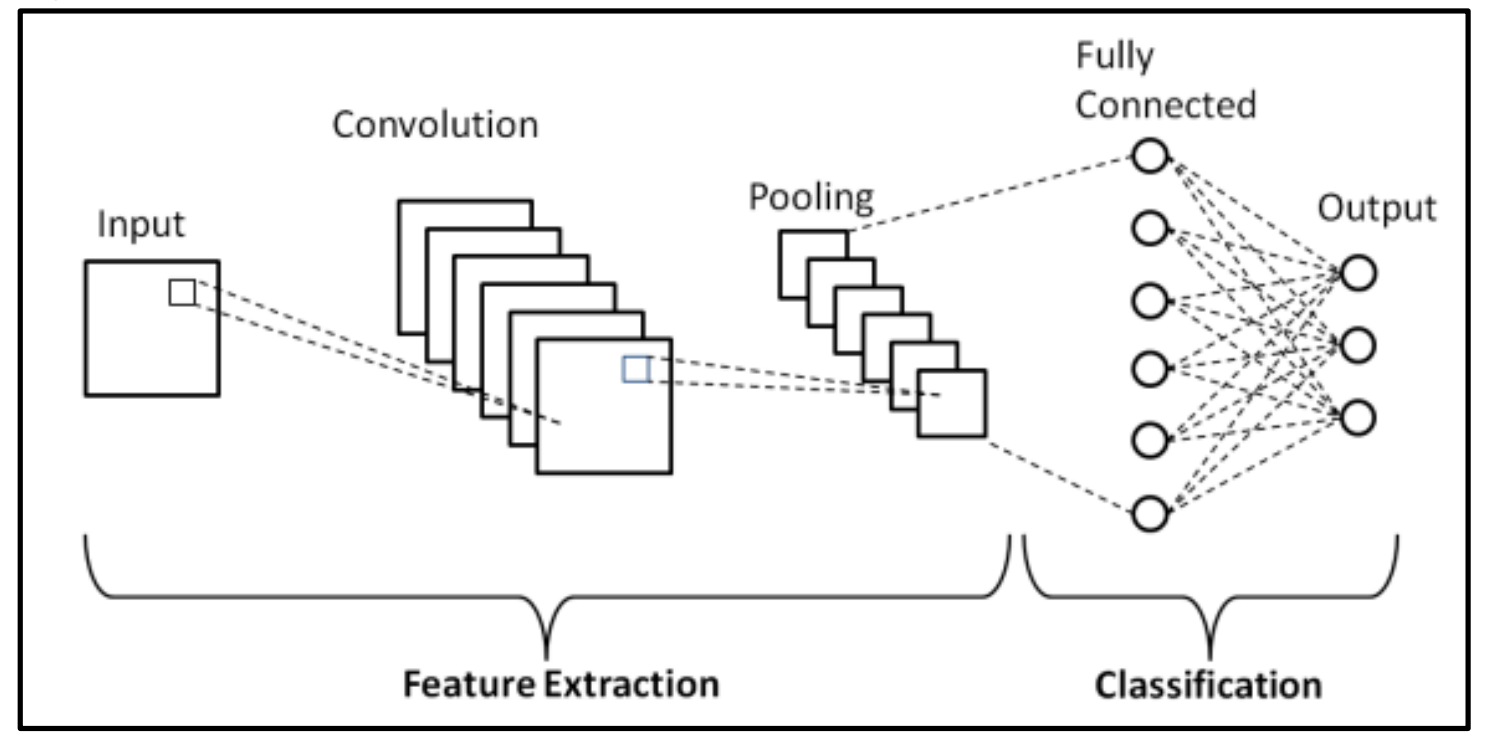

Figure 4: Architecture of convolutional neural network

In our study, we introduce the implementation of 3D-CNN model in details. First, given the volumetric nature of MR images, a network architecture that uses 3D convolutions was developed. The inputs were normalized 3D T1-weighted images and the outputs to be predicted were subject groups.

The architecture of the network contains: 3d-conv input layer with shape $(1,48,96$, 96) and mask $(410,7,7)$ with 50 kernels of size $5 \times 5 \times 5$ with alternating stride $(6,7,7)$ , a Rectified Linear Unit (activation layer); a max pooling $3 \mathrm{~d}$ with size $(6,7,7)$, two dropout layers with 50\% and 50\% respectively, flatten layer, dense layer and dense output layer with softmax activation and three classes: HC, MCI, AD. (See figure 5). 


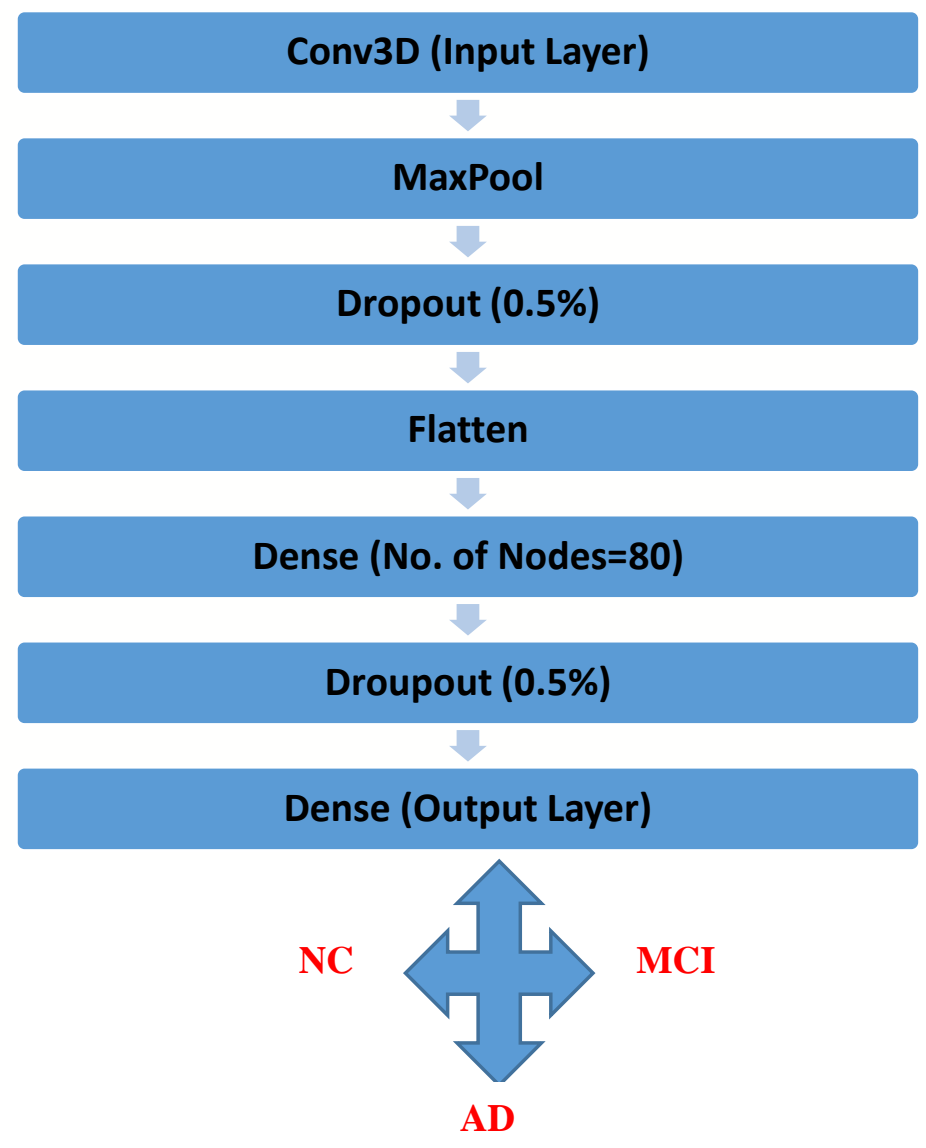

Figure 5: Block Diagram of 3D Convolutional Neural Network

\section{- Network Architecture}

We use the set of learnt weights of each basis of the sparse autoencoder as a 3D filter of a 3D convolutional layer for each basis of the sparse autoencoder we previously trained. We get a convolutional layer of 410 3D feature maps by using all the bases and applying the convolutions. Convolution of an image with a basis gives a feature map of size $(485+1)(965+1)(965+1)=42 \times 90 \times 90$, because the patches are $5 \times 5 \times 5$ in size. We additionally apply a sigmoid activation function to every unit in the feature map and add the bias term associated with the basis. This convolutional layer is anticipated to uncover local patterns and structures in the 3D input image, allowing the algorithm to take advantage of the image's 3D topological and spatial information. Pooling layers come after convolutional layers. We employ max-pooling, which divides each feature map into numerous non-overlapping and contiguous hidden unit neighborhoods. Only the hidden unit with the highest activation is kept in each neighborhood. As previously stated, the pooling process minimizes the number of units in a hidden layer, which is beneficial. Pooling also improves image robustness in the face of minor image distortions like translations. To minimize the size of the feature maps of the convolutional layer, we use a $5 \times 5 \times 5$ max-pooling procedure in our technique. As a result, each feature map becomes a max-pooled feature map with a size of $(42 / 5)(90 / 5)(90 / 5)=8 \times 18 \times 18$. After that, the outputs of each max-pooled feature map are piled. There are a total of $410 \times 8 \times 18 \times 18$ outputs with 410 feature 
maps of size $8 \times 18 \times 18$. These outputs are fed into a three-layer fully-connected neural network as inputs (i.e. with an input, hidden and output layer). We use an 80-unit hidden layer with a sigmoid activation function and a 3-unit output layer with a softmax activation function. The conditional probability that the input belongs to each of the classes are represented by the three units in the output layer (AD, MCI, and HC). Figure 6 demonstrates the network architecture model.

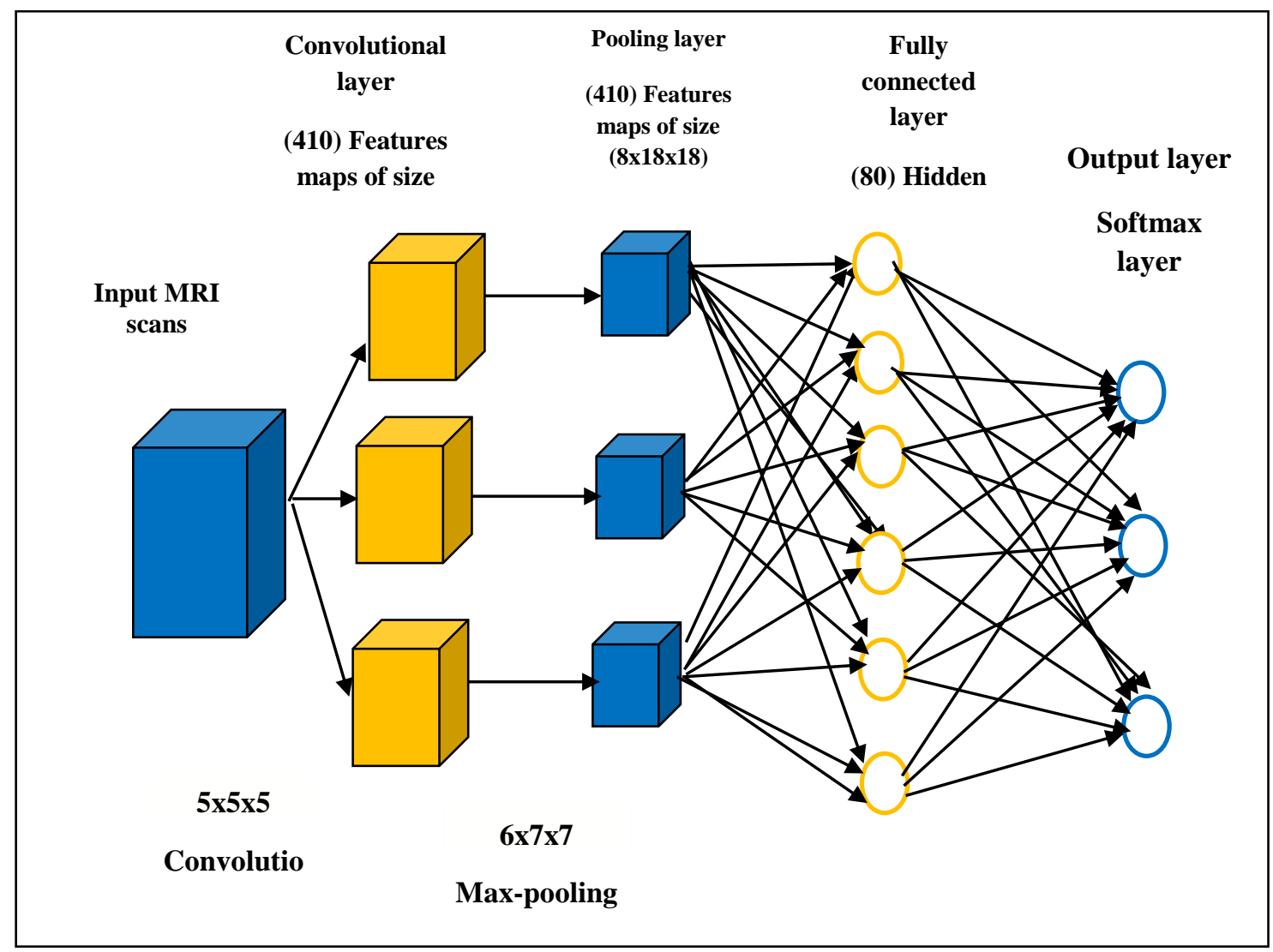

Figure 6: Architecture of the 3D-convolutional neural network used for 3-way classification

\section{Experimental Results and Discussion}

The proposed approach involves two steps. Firstly, the dataset is preprocessed to make it suitable for building our model. After preprocessing, the dataset is then split into train and test sets. Our interest is to obtain the latent representation of the input learned by the SAE model and use it to train the $3 \mathrm{~d}-\mathrm{CNN}$ model. Hence, once the SAE model is trained, the encoding part is used to create another network. The second stage is to train the $3 \mathrm{~d}-\mathrm{CNN}$ classifier using the transformed train set and then make predictions on the test set. The proposed method prevents any possible data leakage and overfitting.

Performance of the 3D CNN was validated and tested on patients and controls, with three classes HC, MCI and AD. The classification included three steps: (i) training with 897 patients with 93\% accuracy (ii) validation with 100 patients with $94 \%$ accuracy and (iii) testing with 300 patients with $87,87 \%$ accuracy as shown in figure 7 and 8 respectively. First, MRI data was randomly split into a large training and 
validation set (90\% of images) and a testing set (10\% of images) using 10 -fold validation. CNN's performance was evaluated by several performance measures, i.e. sensitivity, specificity and accuracy. Sensitivity measures the proportion of true positives correctly identified, whereas specificity refers to the proportion of true negatives correctly identified. The accuracy of a classifier represents the overall

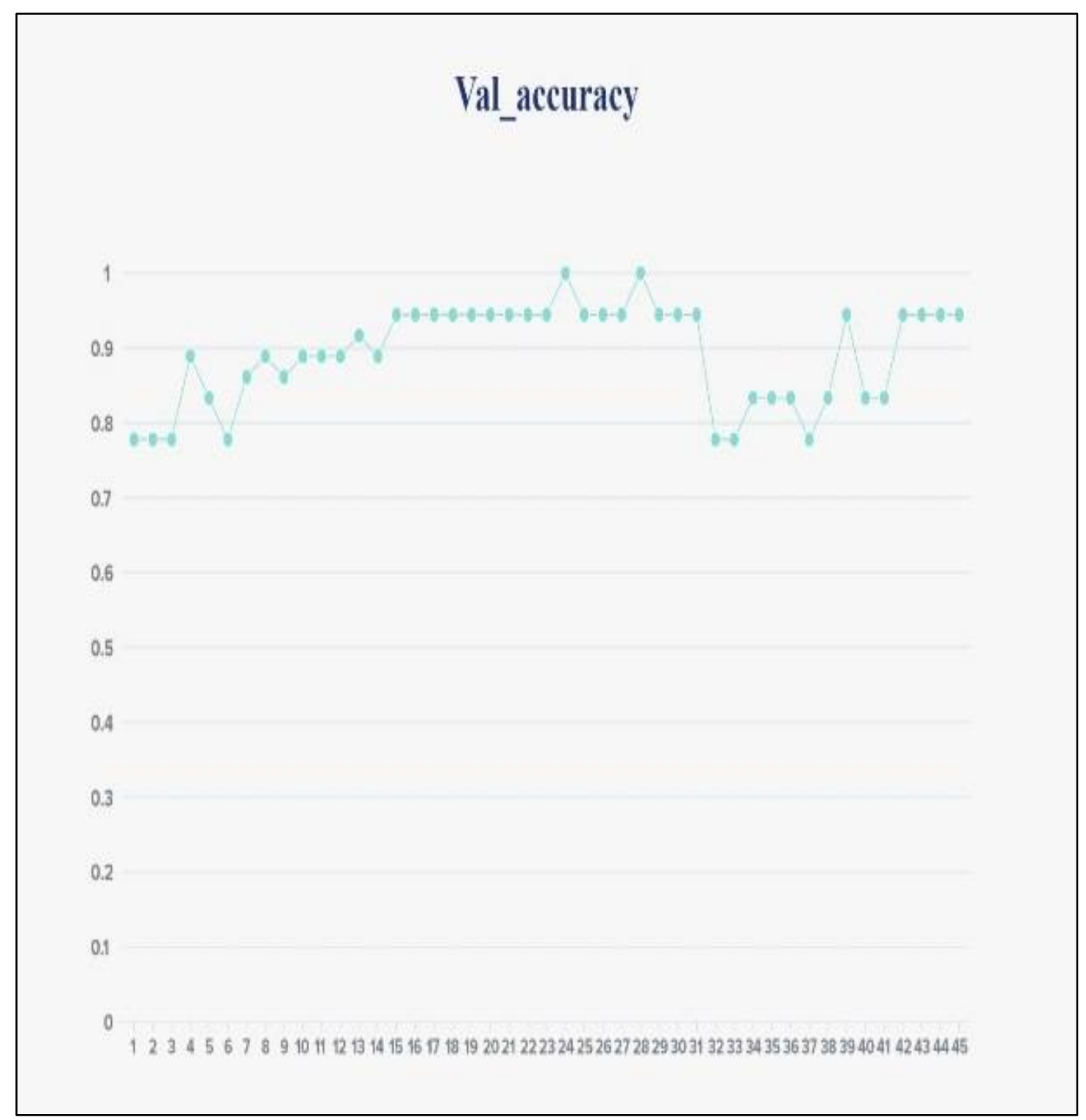

proportion of correct classifications as shown in table 2 .

Figure 7: Validation Accuracy

Table 2: Performance Evaluation of the Proposed Model

\begin{tabular}{|c|c|c|c|}
\hline Class & Precision & Recall & F1-score \\
\hline CN & 0.91 & 0.94 & 0.93 \\
\hline MCI & 0.85 & 0.85 & 0.85 \\
\hline AD & 0.91 & 0.88 & 0.89 \\
\hline
\end{tabular}




\section{Train_accuracy}

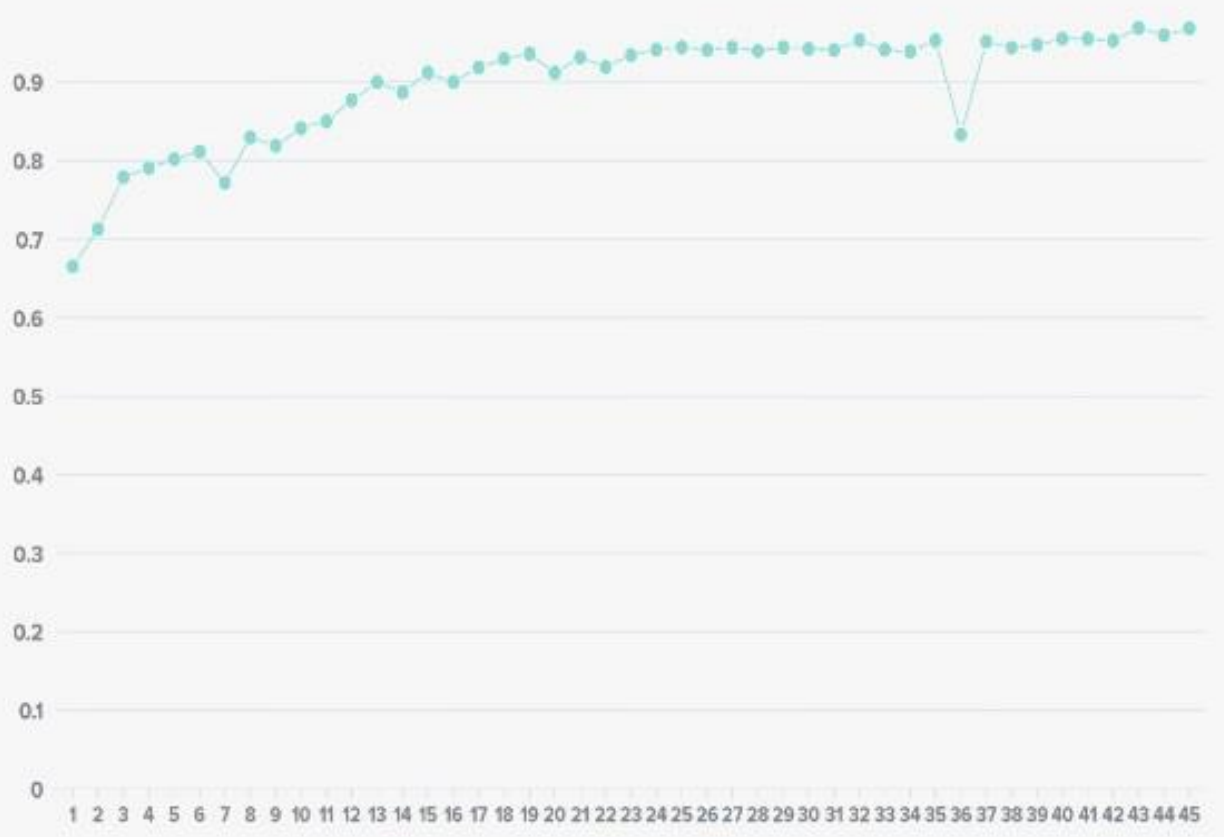

Figure 8: Training Accuracy

The result shows that the low-dimensional features learned by our sparse autoencoder improves the classification performance of the 3D-CNN, since the proposed method performs better than the our previous model which used 3D-CNN without sparse auto encoder, which is a demonstration of the fact that the sparse autoencoder is capable of retaining the information in the input data while obtaining optimal low dimensional features. The model performs well on the test data, which is a major pointer to its efficiency since the model has not previously seen the data. Furthermore, the proposed approach is compared with some recent scholarly works as shown in Table 3 , and it shows better performance than those reported in the literature. 
Table 3: Classification Performance Comparison between the Proposed Model and Other Recent Scholarly Works.

\begin{tabular}{|c|c|c|c|c|c|}
\hline Reference & Methodology & $\begin{array}{c}\text { Way- } \\
\text { classification }\end{array}$ & Accuracy & $\begin{array}{c}\text { Precision } \\
(\%)\end{array}$ & $\begin{array}{c}\text { Recall } \\
(\%)\end{array}$ \\
\hline$[27]$ & $\begin{array}{c}\text { KNN-Decision } \\
\text { tree- Deep learning }\end{array}$ & $\begin{array}{c}\text { 5- way } \\
\text { (CN-EMCI- } \\
\text { LMCI-SMC-AD) }\end{array}$ & $88.24 \%$ & $89 \%$ & $86 \%$ \\
\hline$[\mathbf{2 8}]$ & Deep CNN & $\begin{array}{c}4 \text { way } \\
\text { (AD, EMCI, } \\
\text { LMCI, NC) }\end{array}$ & $73.75 \%$ & - & - \\
\hline$[5]$ & $\begin{array}{c}\text { Deep Learning } \\
\text { methods (sparse } \\
\text { autoencoders and } \\
\text { 3D CNNs) }\end{array}$ & $\begin{array}{c}\text { (AD vs HC, AD } \\
\text { vs MCI, HC vs } \\
\text { MCI) }\end{array}$ & $89.47 \%$ & - & - \\
\hline $\begin{array}{c}\text { Our } \\
\text { approach }\end{array}$ & $\begin{array}{c}\text { Deep Learning } \\
\text { methods (sparse } \\
\text { autoencoders and } \\
\text { 3D CNNs) }\end{array}$ & $\begin{array}{c}\text { 3-ways } \\
\text { (CN,MCI,AD) }\end{array}$ & $87.8 \%$ & $91 \%$ & $88 \%$ \\
\hline
\end{tabular}

From the results obtained so far, it can be grasped that the proposed method shows significant enhancement compared to the other methods in terms of classification performance. And it is clear that the proposed sparse autoencoder improves the accuracy of the CNN compared to a case where the CNN alone was used to make predictions. The results also show that improved performance can be achieved not only by improving the structure of the neural network, but also by improving the preprocessing stage of the classification process.

\section{CONCLUSION and Future Work}

In this paper, we propose an enhanced sparse autoencoder-based 3D-CNN for Alzheimer's disease prediction. The sparse autoencoder was used to learn the optimum data representation, and the $3 \mathrm{D}-\mathrm{CNN}$ was utilized to create predictions based on the learnd records. The SAE was optimized with the Adam method and batch normalization. On test data, the model gave an accuracy $87.8 \%$. In future study, we can combine multiple datasets using advanced deep learning algorithms to boost the efficiency and efficacy of AD prediction at an earlier stage. In addition to, the proposed methodology will test on real data.

\section{References}

[1] Sameer Mahajan, Gayatri Bangar and Nahush Kulkarni, " Machine Learning Algorithms for Classification of Various Stages of Alzheimer's Disease: A review", International Research Journal of Engineering and Technology (IRJET), Volume: 07 Issue: 08 | Aug 2020.

[2] P. Lodha, A. Talele and K. Degaonkar, "Diagnosis of Alzheimer's Disease Using Machine Learning," ,Fourth International Conference on Computing Communication Control and Automation (ICCUBEA), pp. 1-4, DOI: 10.1109/ICCUBEA.2018.8697386, Pune, India, 2018. 
[3] R. Brookmeyer, E. Johnson, K. Ziegler-Graham and H. Arrighi, "Forecasting the global burden of Alzheimer's disease", Alzheimer's \& Dementia, vol. 3, no. 3, pp. 186-191, 2007.

[4] Ahmad Waleed Salehi, Preety Baglat, Gaurav Gupta, " Alzheimer's Disease Diagnosis using Deep Learning Techniques ", International Journal of Engineering and Advanced Technology (IJEAT), Volume-9 Issue-3, February, 2020.

[5] Adrien Payan and Giovanni Montana, " Predicting Alzheimer's disease: a neuroimaging study with 3D convolutional neural networks", arXiv: 1502.02506v1 [cs.CV] 9 Feb 2015

[6] Veitch, D. P., Weiner, M. W., Aisen, P. S., Beckett, L. A., Cairns, N. J., Green, R. C., et al. "Understanding disease progression and improving Alzheimer's disease clinical trials recent highlights from the Alzheimer's disease neuroimaging initiative", Alzheimers Dement 15, 106-152. doi: 10.1016/j.jalz.2018.08.005, 2019.

[7] Taeho Jo, Kwangsik Nho and Andrew J. Saykin, "Deep Learning in Alzheimer's Disease: Diagnostic Classification and Prognostic Prediction Using Neuroimaging Data", Frontiers in Aging Neuroscience, Volume 11 | Article 220, 2019.

[8] Iago Richard Rodriguesda Silva, Gabriela dos Santos Lucas eSilva and Rodrigo Gomesde Souza, " Chapter four - Deep learning for early diagnosis of Alzheimer's disease: a contribution and a brief review", Deep Learning for Data Analytics Foundations, Biomedical Applications, and Challenges, ScienceDirect, Pages 63-78 , 2020.

[9] Ibomoiye Domor Mienye, Yanxia Sun and Zenghui Wang, "Improved sparse autoencoder based artificial neural network approach for prediction of heart disease", Informatics in Medicine Unlocked, Volume 18, 2020.

[10] Debesh Jha andGoo-Rak Kwon, " Alzheimer's Disease Detection Using Sparse Autoencoder, Scale Conjugate Gradient and Softmax Output Layer with Fine Tuning", International Journal of Machine Learning and Computing, Vol. 7, No. 1, February 2017.

[11] Jyoti Islam and Yanqing Zhang, " Understanding 3D CNN Behavior for Alzheimer's Disease Diagnosis from Brain PET Scan", arXiv:1912.04563v1, 10 Dec 2019.

[12] Islam, J., Zhang, Y., "Early Diagnosis of Alzheimer's disease: A Neuroimaging Study with Deep Learning Architectures." Proceedings of the IEEE Conference on Computer Vision and Pattern Recognition Workshops. 2018.

[13] Islam, J., Zhang, Y., "An Ensemble of Deep Convolutional Neural Networks for Alzheimer's Disease Detection and Classification." arXiv preprint arXiv: 1712.01675 (2017).

[14] LeCun Y., Jackel L. D., Bottou L., Cortes C., Denker J. S., Drucker H., et al. "Learning algorithms for classification: A comparison on handwritten digit recognition," in Neural Networks: The Statistical Mechanics Perspective eds Oh J. H., Kwon C., Cho S. (Singapore: World Scientific; ) 261-276, 1995.

[15] Yechong Huang1, Jiahang Xu1, Yuncheng Zhou1, Tong Tong2 , Xiahai Zhuang1 and the Alzheimer's Disease Neuroimaging Initiative (ADNI), " Diagnosis of Alzheimer's Disease via Multi-Modality 3D Convolutional Neural Network", 
frontiers in Neuroscience, volume 13, Article 509, doi: 10.3389/fnins.2019.00509, 2019.

[16] Suk HI, Lee SW, Shen D, A. S. D. N. "Initiative. Deep sparse multi-task learning for feature selection in Alzheimer's disease diagnosis" Brain Struct Funct. ;221(5):2569-87, 2016.

[17] Tao S, Zhang T, Yang J, Wang X, Lu W. "Bearing fault diagnosis method based on stacked autoencoder and softmax regression". In: Control conference (CCC), 34th Chinese, IEEE. 2015. pp. 6331-5, 2015.

[18] Suk H-I, Lee S-W, Shen D, A. S. D. N. Initiative. "Deep ensemble learning of sparse regression models for brain disease diagnosis". Med Image Anal.;37:101-13, 2017.

[19] Ehsan Hosseini-Asl, Robert Keynton and Ayman El-Baz, " ALZHEIMER'S DISEASE DIAGNOSTICS BY ADAPTATION OF 3D CONVOLUTIONAL

NETWORK", International Conference on Image Processing (ICIP), Published in the IEEE, 2016.

[20] Firouzeh Razavi, Mohammad Jafar Tarokh and Mahmood Alborzi, "An intelligent Alzheimer's disease diagnosis method using unsupervised feature learning", Journal of Big Data, published at springer, doi.org/10.1186/s40537-0190190-7, 6:32, 2019.

[21] K.R. Kruthika, Rajeswari, H.D. Maheshappa, Alzheimer's Disease Neuroimaging Initiative, " CBIR system using Capsule Networks and 3D CNN for Alzheimer's disease

diagnosis", Informatics in Medicine Unlocked, 59-68, Science Direct, ELSIEVER, 2019.

[22] Debesh Jha and Goo-Rak Kwon," Alzheimer's Disease Detection Using Sparse Autoencoder, Scale Conjugate Gradient and Softmax Output Layer with Fine Tuning", International Journal of Machine Learning and Computing, Vol. 7, No. 1, February 2017.

[23] Xiuli Bi, Shutong Li, Bin Xiao, Yu Li, Guoyin Wang and Xu Ma, "Computer Aided Alzheimer's Disease Diagnosis by An Unsupervised Deep Learning Technology", Neurocomputing journal, DOI: 10.1016/j.neucom.2018.11.111, 2019.

[24] Husnu Baris Baydargil, Jang-Sik Park, Do-Young Kang, "Classification of Alzheimer's Disease Using Stacked Sparse Convolutional Autoencoder", 19th International Conference on Control, Automation and Systems (ICCAS), Korea (South), IEEE, DOI: 10.23919/ICCAS47443.2019.8971696, 2019.

[25] Francisco J. Martinez-Murcia, Andres Ortiz, Juan-Manuel Gorriz, Javier Ramirez, and Diego Castillo-Barnes, "Studying the Manifold Structure of Alzheimer's disease: A Deep Learning Approach Using Convolutional Autoencoders", IEEE JOURNAL OF BIOMEDICAL AND HEALTH INFORMATICS, VOL. 24, NO. 1, JANUARY 2020

\section{[26] WWW.ADNI-INFO.ORG}

[27] Muhammad Shahbaz1, Shahzad Ali2 a , Aziz Guergachi3, Aneeta Niazi1 and Amina Umer," Classification of Alzheimer's Disease using Machine Learning Techniques", - 8th International Conference on Data Science, Technology and Applications, DATA, 2019. 
DIAGNOSIS OF ALZHEIMER'S DISEASE BY THREE-DIMENSIONAL CONVOLUTIONAL NEURAL NETWORK USING UNSUPERVISED FEATURE LEARNING METHOD

[28] J. Islam and Y. Zhang, "A novel deep learning based multi-class classification method for Alzheimer's disease detection using brain MRI data," in International Conference on Brain Informatics, 2017, pp. 213-222: Springer 